required in different azimuths to ascertain that we are dealing with a single circular disc, and not with two neighbouring discs, as in the case of Capella. The resulting angular diameter is $0.045^{\prime \prime}$; Prof. Eddington had predicted $0.05 \mathrm{I}^{\prime \prime}$ from the visual magnitude and assumed surface brightness, and Prof. Seeliger deduced $0.042^{\prime \prime}$ in a somewhat similar manner. The linear diameter found (about 300 times that of the sun) depends on the assumed parallax, which, unfortunately, is decidedly uncertain; further determinations seem urgently to be called for. Prof. Lindemann pointed out the extraordinarily low density that such a diameter implies if we assume that the mass is of the order of twenty-five times that of the sun.

Other stars for which a diameter determination is hopeful are Antares and Aldebaran, and possibly Arcturus. Sirius and Vega will doubtless be attempted, but with less prospect of success.

A. C. D. C.

\title{
Culture and Environment in the Cameroons.
}

$\mathrm{FEW}$ areas in the African continent present problems of greater interest to the anthropologist than the Cameroons. Although the Germans produced a considerable amount of literature relating to the area while it was under their rule, there is still a great deal of work to be done before the complex ethnology of the country is elucidated. Capt. L. W. G. Malcolm, who saw service in the Cameroons during the war, is preparing a monograph which will be one of the first-fruits of our occupation. At a recent meeting of the Royal Anthropological Institute he gave a preliminary account of certain questions connected with the distribution of types of culture and its relation with the geographical environment.

In the Cameroons there are three main racial stocks, namely, the Bantu-speaking tribes, the Sudanese, and the Pygmies. Subsidiary immigrant races occupy certain areas in the north-east. Between the Bantu-speaking tribes and the Sudanese there are a number of tribes, some of which do not speak Bantu languages, and there is a distinct boundary between the Bantu-speaking and Sudanese races which is determined solely by the geographical nature of the country.

The material culture of the grassland area reveals the fact that the problem is of a most complex character. When dealing with tribes of mixed affinities it is extremely difficult to determine the various strata. Not only is there a local mixing of the tribes, but there are also various elements which have been brought in by invading tribes. This is partisularly the case in Bagam, where the tribe has been in- fluenced particularly from Bamum, in the north-east, while from Babanki, in the north, various forms of iron weapons have been introduced. The Balis have introduced among the grassland tribes the sleeveless gowns worn by the men. After Hausa and Fulani elements and the influence of the forest-beit tribes have been eliminated, it would appear that the chief characteristics of the grassland culture are weapons of copper, iron, and brass; socketed spearheads; a simple bow made from raphia palm, with a flat bowstring; arrows with wooden points; shields, either plaited or reinforced with wood; wooden slit-gongs; drums with skin tympana and wooden tautening wedges; flanged iron bells; iron-working (smelting and smithing); brass castings (Bagam and Bamum); pottery (coiled in the north-east); jutan cloth and woven fibre; decorative art with triangles and zigzags; cicatrisation; filing or chipping of the incisors; smoking pipes of metal and clay; animal and ancestral cults; and the use of carved masks and images. One of the most obvious and striking peculiarities of the grassland culture, however, is the quadrangular hut, with pyramidal or conical roof, ranged in streets, which differs distinctively from the forest-belt hut standing in its own irregularly placed clearing.

Capt. Malcolm's careful analysis of the culture of one area only of the Cameroons, even in this preliminary form, was not merely an indication of the extremely interesting material which still awaits investigation; it was also a valuable object-lesson of the method of studying a backward population which should form the essential basis of our administration in this and similar areas.

\section{The Science Masters' Association.}

$A^{T}$ the invitation of the Board of the Faculty of Natural Science, the annual meeting of the Science Masters' Association was held in Oxford on January $4-7$. About two hundred members attended, and by the kindness of the Master of Balliol and the President of Trinity they were housed in these two colleges.

The meeting began on the evening of January 4 with the address of the president (Mr. A. Vassall, of Harrow) on "Some Aspects of Science and Education." Mr. Vassall dealt with education in science from the preparatory school to the university, and showed the evils of teaching in the earlier stages as if all the boys were ultimately to become specialists. The teaching should be such as to give every boy an opportunity of realising the scope and aims of science. It should impart to every boy the understanding of scientific problems necessary for the equipment of a modern State, and not be merely a training for future work in science, which in many cases will never be taken up seriously.

A lecture on spectroscopy, given by Prof. T. R. No. 2673 , VOL. IO6]
Merton, was concerned largely with the part played by observation and technique; observation cannot be effective with faulty apparatus. In the spectroscopy of gases the influence of traces of impurities is very great, and many beautiful experiments were shown to illustrate the methods of dealing with them. The conditions under which nitrogen gives a band spectrum and a line spectrum were shown; a tube was exhausted and filled with helium sufficiently free to enable the conditions for obtaining either a band spectrum or a line spectrum to be demonstrated. Also, a striking experiment was shown by which the presence of neon in the atmosphere was made evident. The method of getting hydrogen into and out of tubes by means of a heated side-tube of palladium was illustrated, and the curious fluorescence of parts of the human body was shown by illuminating the audience by light of wave-length about $3660 \AA$.U., obtained by using a quartz mercury vapour lamp and a screen of special glass devised by Prof. R. W. Wood.

There were demonstrations in the various University laboratories, illustrating much of the teaching 